\title{
THE STELLAR VARIABILITY FROM HIPPARCOS PHOTOMETRY
}

\author{
M. GRENON \\ Geneva Observatory \\ 1290 Sauverny, Switzerland
}

\section{Introduction}

During the development of the Hipparcos satellite, the opportunity to use the star mapper to perform a whole sky two colour intermediate accuracy photometric survey, and the main-mission detector for high accuracy photometry of target stars was identified. The instrument was optimised and the pre-launch calibrations performed to pre-determine the pass-bands with an accuracy sufficient to cope, in-orbit, with the foreseen aging of the detection chains. Photometry became an important by-product of the mission, very complementary to the astrometric results. It was the first opportunity to monitor the sky during 3 - 4 years without selection biases and with a precision similar to that achieved from the ground with the best classical techniques. A systematic detection of small amplitude variables was the expected return.

\section{The correction of the aging effect}

The radiation by cosmic and solar particles induced transmission losses mainly in the green to violet part of the spectrum. Since the $\mathrm{Hp}$ band is broad, $\lambda \lambda 380-900 \mathrm{~nm}$, the effect was a chromatic change of the band, and hence of the Hp magnitude as large as 0.6 and 0.2 mag over the mission for $\mathrm{V}-\mathrm{I}=0$ and $7 \mathrm{mag}$ respectively. Photometric reductions were performed twice a day using a subset of the 29000 standard stars. Zero-point errors on the magnitude scale were then less than 0.001 mag for each data set. Correction of chromatic drifts requires the knowledge of the star colour; consequently error on it induces a linear trend in the Hp time series.

\section{The variability detection threshold}

The Hipparcos photometry is essentially photon-noise limited. The error on Hp per transit are 3 , $5,8,15$ and 33 mmag for $\mathrm{Hp}=3,5,7,9$ and $11 \mathrm{mag}$. The mean number of useful transits, function of the galactic latitude, is 110 , with a range of 20 to more than 300 . The time sampling is peculiar with groups of 4 to 6 transits separated by 20 and $108 \mathrm{~min}$, repeated 3 to 5 weeks later, an interval depending on the scanning law. This sampling limits the range of detectable periods and introduces severe aliasing problems. Several algorithms developed at Geneva and RGO, allow to classify stars as non-variable, suspected or variable with thresholds depending on the magnitude and the number of transits cf. Eyer et al.(1997), van Leeuwen (1997). The detectable amplitude for variables are $0.8,20$ and $120 \mathrm{mmag}$ for $\mathrm{Hp}=4,8$ and 12 . Known variables as Ap or BY Dra type may escape the detection if faint, whereas on the brighter side, stars previously accepted as constant appeared variable because of the higher accuracy and the large number of measurements.

\section{The variability analysis}

According to statistical tests about 11600 stars are variable or possibly variable at the $0.1 \%$ error level. When the ratio of intrinsic scatter (corrected for photon and reduction noises) over the noise exceeds a threshold leaving less than few percent spurious periods, a search for signal periodicity was undertaken. Some 3343 stars were not investigated. A careful analysis of instrumental effects due to mispointing, image overlap, high-background, allowed to clean the time series and remove cases mimicking flares or eclipses. Abbe test was used to detect long term trends true or due to errors on 
the colour. A series of algorithms optimised for specific light-curve shapes, Fourier transform for symmetric distributions, Renson for possible eclipsing binaries etc. were applied to the final time series. Period search was an iterative process, followed by a semi-automatic curve fitting leading to the period, epoch, light-curve extrema and residuals for all periodic variables. Solutions from Geneva and RGO were then carefully cross-checked and compared to literature data when available at RGO. Due to the time sampling, only the first mode of multiperiodic stars is detectable from Hipparcos data.

\section{The Catalogues and Atlases}

The data relative to periodic variables, as described in the Guide to the data (ESA 1997), are given in the Hipparcos Variability Annex Part 1. Among 2712 periodic variables, 951 are new discoveries and other 118 were previously NSV suspected variables. 171 solutions were found by combining Hipparcos data with literature information. Folded light-curves are displayed in part A of the Atlas with super-imposed fitted curves in 2287 cases (1189 from Geneva and 1098 from RGO).

The catalogue of unsolved variables (Annex Part 2) contains 5542 entries; among them 1123 were already known variables and 233 were NSV stars. 4086 variables are new and for 1953 of them, the variability status was clear enough to have a star name allocated by the Samus' team at Moscow. About 3000 unsolved variables have still no variability type. For unsolved variables an estimation of the peak to peak amplitude as described by Eyer et al.(1997) is provided. The best defined light-curves are displayed in part $\mathrm{C}$ of the Atlas.

Large amplitude irregulars were monitored by AAVSO observers prior and during the mission. The transformation of visual magnitudes into Hp allowed to construct combined light-curves for 274 LPV's and irregulars, with a high time coverage, displayed in part B of the Atlas.

\section{New variables}

The most frequent new periodic variables are EB, then EA eclipsing binaries, followed by SR and SPB. Eclipsing are mainly B to early G type stars with one component somehow evolved. A bias against long period eclipsing binaries is present. The number of SPB's was increased by a factor 10 . $\mathrm{ACV}$ and $\delta$ Sct variables are the next most frequently detected new variables. Periods were rarely found for multiperiodic main sequence stars nor for late type rotating stars.

Among 4086 new unsolved variables, only 1134 have a variability type. The most frequent types for new variables are SR, I, L then $\gamma$ Cas. Many B and A stars were classified as $L$ type, waiting for a spectroscopic improvement of their type.

\section{The Variability across the HR-diagram}

The fraction of variables in the HR-diagram was estimated for parallax stars, showing all red giants to be variable with amplitudes anticorrelated with Teff and surface gravity. Early type stars appear nearly all variable, especially if luminous or B2-B3 main sequence stars. The low main sequence looks constant because the amplitudes are below the detection limit, a simple magnitude effect. Distributions of periods and amplitudes across the HR-diagram are now available.

The distribution of the intrinsic scatter, in bins of spectral sub-type and luminosity class, provides quantitative information on the mean amplitude and its range across the HR-diagram for all stars. Islands of stability are found on both sides of the instability strip: the first from B9 to A7 stars, narrowing towards high luminosity and the second from F2 to G5 dwarfs up to G5 to K2 giants. The level of microvariability across the whole HR-diagram allows to select targets for further study as asteroseismology, or constant comparison stars. The new limits of gaps and instability strips are direct tests of the stellar evolution models.

\section{References}

Eyer L., Grenon M., 1997, 'Hipparcos Venice' 97', ESA SP-402, 462-473

van Leeuwen F., 1997, 'Hipparcos Venice' 97', ESA SP-402,19-24

ESA, The Hipparcos and Tycho Catalogues, 1997, ESA SP-1200 